\title{
Validation test on real scale UV reactor for ballast water treatment
}

\author{
Jiyong $\mathrm{Joo}^{{ }^{\dagger}}$, Gilju Jung${ }^{2}$, Ilseok $\mathrm{Oh}^{3}$, Taejin Rhee $^{2}$ \\ ${ }^{1}$ Advanced Research Center, Korea Shipbuilding \& Offshore Engineering (KSOE), 55, Bundang-ro, Bundang-gu, Seongnam-si, Gyeonggi-do, Korea \\ ${ }^{2}$ Engineering Center BWTS, Hyundai welding, 641, Jongga-ro, Jung-gu, Ulsan, Korea \\ ${ }^{3}$ Engine and Machinery department, Hyundai Heavy Industries (HHI), 1000, Bangeojinsunhwan-doro, Dong-gu, Ulsan, Korea
}

\section{ABSTRACT}

Design of UV reactors for drinking water has been widely studied using computational fluid dynamics (CFD) modeling. When combined with collimated beam test (CBT), CFD becomes a powerful tool for verifying the performance of a UV reactor. However, the design of real scale UV reactor for ballast water treatment system (BWTS) has not been extensively studied due to their relatively short R\&D history. For this reason, we have attempted to validate the UV reactor through the results of the biological efficacy (BE) test with Tetraselmis microorganism. Through $\mathrm{BE}$ tests, we found that 3-log Tetraselmis inactivation was satisfied when the reduction equivalent dose (RED) was higher than that of $1,500 \mathrm{~J} / \mathrm{m}^{2}$.

Keywords: Ballast water treatment system (BWTS), Biological efficacy (BE) tests, Collimated beam test (CBT), Computational fluid dynamic (CFD), Reduction equivalent dose (RED), UV disinfection

\section{Introduction}

Ballast water is transferred by vessels to ensure stability during the voyage. Ballasting and deballasting operations occur to offset changes of vessel displacement associated with unloading and loading, respectively. However, the various biological substances contained in ballast water can impact on the native species and cause ecological change in the ocean.

To combat the problem of invasive species from ballast water, the International Marine Organization (IMO) has implemented requirements to treat ballast water prior to discharge [1]. Acceptable concentration of the organism class defined by greater than or equal to $50 \mu \mathrm{m}$ corresponds to zooplankton is less than 10 viable organisms $/ \mathrm{m}^{3}$. Whereas the organism class defined by less than $50 \mu \mathrm{m}$ and greater than or equal to $10 \mu \mathrm{m}$ corresponds to phytoplankton is less than 10 viable organisms/mL [2-3]. Therefore, ballast water treatment system must be designed to properly control the population of these organism classes in treated water.

As indicated in IMO's 2015 final report, among those approved and commercially available ballast water treatment system (BWTS) used for reducing organisms concentration in ballast water, electrolytic disinfection devices in almost $40 \%$, ultraviolet (UV) irradiation devices in $32 \%$, and the use of liquid hypochlorite are found in almost $17 \%$ of systems [4-6]. Among commercially available BWTS,
UV technology has a number of distinct advantages since it does not produce by-products that can be harmful to humans or aquatic life. In addition, UV disinfection is an eliminating the need to generate, or store toxic/hazardous chemicals. Therefore, the UV disinfection has been considered to be an attractive alternative to chlorine. In order to install BWTS on vessel with UV disinfection devices, it is necessary to think the UV disinfection level and operation cost. And these factors are highly dependent on UV reactor geometry configuration [7-8]. As far as we know, however, papers on the design of real scale UV reactor for BWTS have not been extensively published because environmental regulations on ballast water discharge have been recently established. Cosman et al. [8], in 2010, demonstrated the validation protocol for performance test by aid of CFD using the virus (i.e., MS2 Phage) in order to apply to BWTS. However, no validation test using phytoplankton under a real scale UV reactor was systematically conducted. It is crucial to investigate the inactivation behaviors in the 10-50 $\mu \mathrm{m}$ size class in challenge water since most commercially available BWTS filters theoretically remove organisms greater than or equal to $50 \mu \mathrm{m}$.

In this manuscript, we demonstrate the design of real scale UV reactor for BWTS by aid of CFD. Furthermore, a large scale $\mathrm{BE}$ tests using the phytoplankton as target organism were performed in order to validate the performance of UV reactor.
This is an Open Access article distributed under the terms of the Creative Commons Attribution Non-Commercial License (http://creativecommons.org/licenses/by-nc/3.0/) which permits unrestricted non-commercial use, distribution, and reproduction in any medium, provided the original work is properly cited.

Copyright (C) 2021 Korean Society of Environmental Engineers
Received November 21, 2019 Accepted February 06, 2020

${ }^{\dagger}$ Corresponding author

Email: jiyong.joo@ksoe.co.kr

Tel: +82-31-210-9580 Fax: +82-31-210-9691

ORCID: 0000-0002-8401-4121 


\section{Experimental Section}

\subsection{Collimate Beam Test (CBT)}

In general, natural concentration for performing the Land Based Testing (LBT) are too low to meet intake requirements $(>10,000$ organisms/mL: phytoplankton) [9] so standard test organism (i.e., Tetraselmis) was added [10]. Tetraselmis is ubiquitous in the marine environment and they are relatively easy to culture and assay [11]. For this reason, Tetraselmis as target organism is suitable for validation of the UV reactor [12-14]. In this study, CBT result (Refer to Fig. S1) which was carried out by [15] was adapted for the numerical analysis. We will discuss it later in detail.

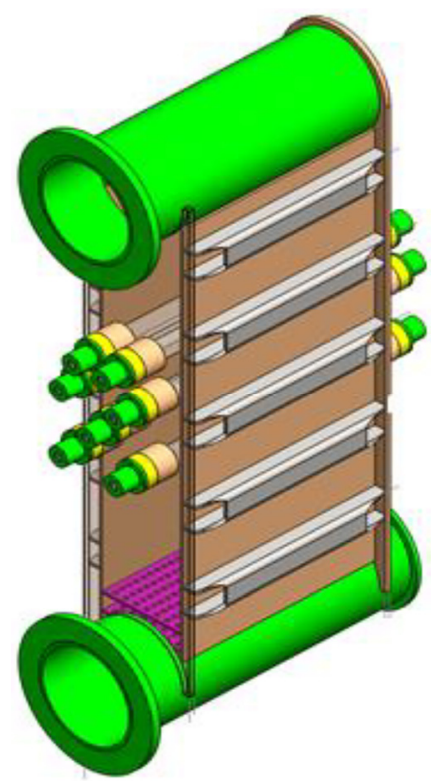

Fig. 1. Configuration of UV reactor.

\subsection{Model}

As shown in Fig. 1, UV reactor is composed of a rectangular channel and two nozzles connecting to ballast water pipelines. UV lamps are located to be perpendicular to the flow direction. And UV reactor contains 7 medium-pressure UV lamps (emitting UV-A, -B, and -C light) which are covered with quartz sleeves to protect the lamps. Each lamp power and efficiency are 8,250 W and 12\%, respectively. Lamp aging factor including quartz transmittance at the end of lamp life is 0.67. And UV transmittance (UVT) is calculated as Eq. (1).

$$
\mathrm{UVT}(\%)=100 \times 10^{-\mathrm{AL}}
$$

Where UVT(\%) is UV transmittance, A and L is absorption coefficient and length (for a $1 \mathrm{~cm}$ ), respectively. For the CFD calculation, UVT is rearranged as Eq. (2).

$$
\mathrm{A}=-(\log U V T[\%] / 100) \times 100
$$

The parameters were selected for the actual seawater characteristics as shown in Table S3.

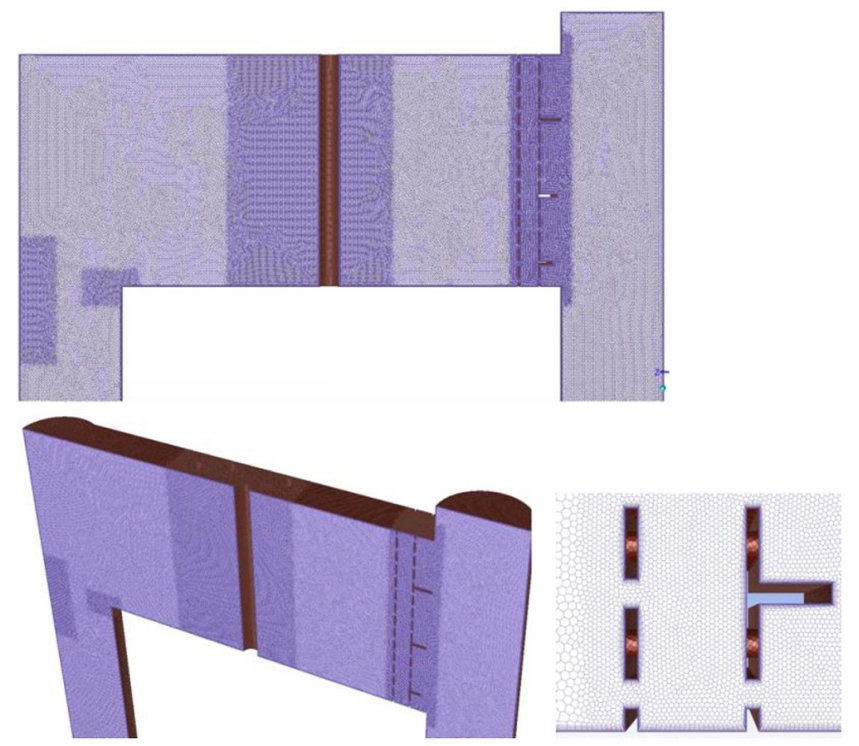

Fig. 2. Computational grid of the UV reactor.

\subsection{Numerical Analysis}

The grid used in this study was created using Ansys Fluent. The grid used in the analysis is shown in Fig. 2. All analysis domains consisted of poly-hedra mesh. About 9.39 million polyhedra cells were created with 10 inflation layers on wall side to detect the boundary layer flow. The minimum size of grid was $1.7 \mathrm{~mm}$ and the maximum size was $4.5 \mathrm{~mm}$. To satisfy the $\mathrm{Y}+$ value of 10 or less, the $1^{\text {st }}$ layer thickness was determined to be $0.1 \mathrm{~mm}$. The grid on UV lamp side, perforated plate and other separation zone was concentrated to better capture the flow nearby.

Realizable k- $\varepsilon$ model was applied to calculate flow field including two perforated plates. This model is an improved model to better predict the size of the vortex in standard $\mathrm{k}-\varepsilon$ model. It includes modified transport equation for the dissipation rate and is derived based on exact transport equation of the mean-square vorticity fluctuation. And the enhanced wall treatment is used for the near-wall modeling because it blends linear and logarithmic laws. The boundary conditions of the model are shown in Fig. 3 and Table S4. Note that symmetrical models are used for CFD simulation in order to reduce the computational cost and convergence time. At all walls, no-slip conditions were applied for velocity and turbulence. Inlet conditions were applied to the mass flow rate, and outlet conditions were applied to $0 \mathrm{~Pa}$. To assume a fully developed flow, the length of the inlet and outlet was extended to 10 times the diameter.

Euler-Lagrangian particle tracking method is used to estimate the dose. The particle trajectories are computed individually at specified intervals during the fluid phase calculation. The particle are uniformly injected into the inflow and a user defined function of Fluent is applied to update the particle scalar (particle dose) whenever the particle position changes. The particle dose was calculated by following formula.

Particle dose $\left(\mathrm{J} / \mathrm{m}^{2}\right)=$ Incident radiation $\left(\mathrm{W} / \mathrm{m}^{2}\right) \times$ Exposure time $(\mathrm{s})$ 


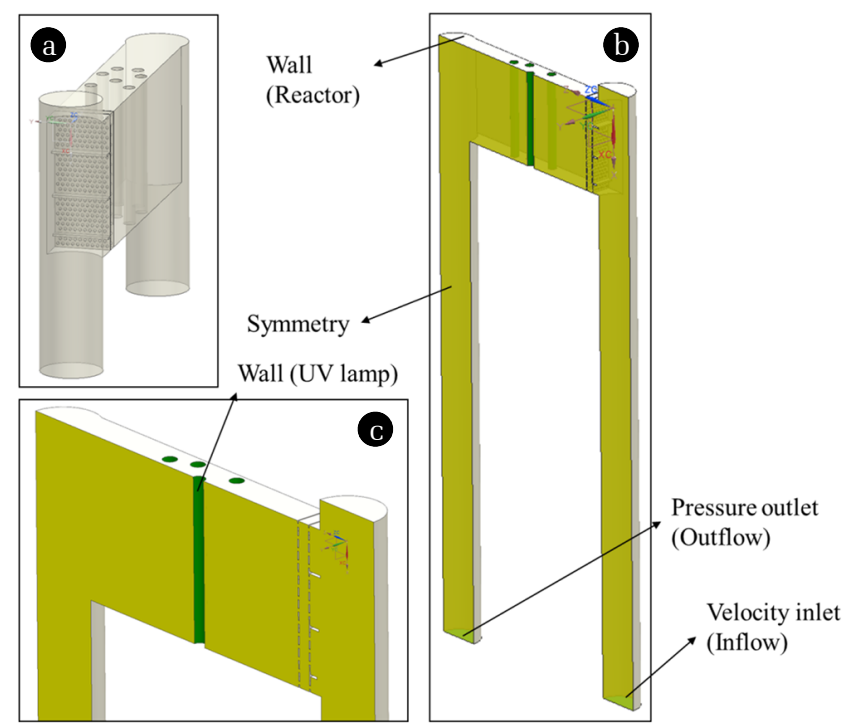

Fig. 3. (a) Fluid domain of the UV reactor, (b), (c) Boundary conditions of the CFD simulation.

\subsection{Land-based Test (LBT) Setup}

In order to perform U.S. Coast Guard (USCG) and IMO LBT tests, challenge water was obtained from the sea near the Korea Marine Equipment Research Institutes (KOMERI) and HHI site, respectively. BWTS was operated using a combination of filtration followed by UV irradiation. The filtration stage aims to remove organisms and suspended matter from ballast water, limited size classes above $50 \mu \mathrm{m}$. The filter volume used for all the tests is $425 \mathrm{dm}^{3}$. Both IMO and preliminary LBT are carried out where flow rate is $200 \mathrm{~m}^{3} / \mathrm{h}$, power is between $\mathrm{Abt}$. 50 to $100 \%$. And target UVT is adjusted by addition of starch and glucose. On the other hands, USCG LBTs are performed where flow rate is Abt. $360 \mathrm{~m}^{3} / \mathrm{h}$, power is fixed at $50 \%$. And UVT is adjusted identical to IMO LBT. In order to investigate the disinfection performance of UV reactor, target microorganism should be designated. According the ETV protocol [9], to be valid, a LBT must be initiated with $>10,000$ organisms $/ \mathrm{mL}$ in the $10-50 \mu \mathrm{m}$ size class in challenge water. As mentioned earlier, natural concentration is too low to meet intake requirements. Herein, Tetraselmis was added as standard test organism on the basis of the ETV protocol [11-13]. For this reason, Tetraselmis was selected as a target microorganism for CFD calculation. Initial organism concentrations varied with test cycle. The minimum value was 1,079 organisms $/ \mathrm{mL}$, the maximum value was 2,955 organisms/mL, and the median value was 1,350 organisms $/ \mathrm{mL}$. The key design parameter of the UV reactor focused on killing ratio of $99.9 \%$ of Tetraselmis target organism. Remind that it requires $99.9 \%$ inactivation ratio to reduce the organism concentration from 10,000 to 10 organisms $/ \mathrm{mL}$. Challenge water was treated twice by UV lamps when ballasting and deballasting. The holding time storing treated water in treated tanks was $5 \mathrm{~d}$. After $5 \mathrm{~d}$, the number of living microorganism at outlet of the treated tank was measured by vital staining method. In addition, the organism concentration is calculated in control discharge water, which is kept similar to the challenge water but has bypassed

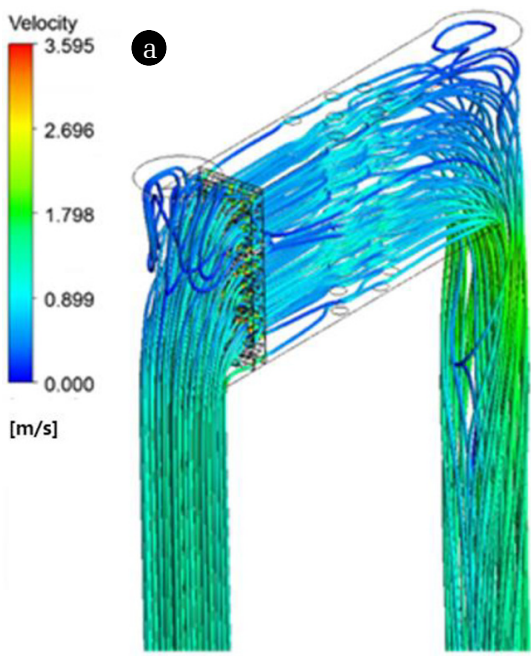

(b)

Fig. 4. (a) Streamline and (b) Flow velocity profile at cross-section of UV reactor.

the BWTS. The quality of the control discharge water provides information on the effect of the holding period on organism concentration to ensure that elimination of organism is caused by the BWTS and not environmental conditions (Refer to the Fig. S2) [9-10].

\section{Results and Discussion}

We recall, as shown in Fig. S1, in order to meet the 3-log inactivation ratio (99.9\% inactivation ratio), we figured out that the required dose is $1,500 \mathrm{~J} / \mathrm{m}^{2}$. However, we set the required dose is $750 \mathrm{~J} / \mathrm{m}^{2}$ since ballast water is treated twice at both ballasting and deballasting. Furthermore, we found that UV-dose response curve follows the first order kinetics until 1,500 J/m². Consequently, we can attain the equation as follow.

$$
\log \left(\mathrm{N}_{0} / \mathrm{N}\right)=(1 / 500) \text { UV dose }
$$

Where,

$\mathrm{N}_{0}$ : Concentration of infectious microorganisms before exposure to UV light

$\mathrm{N}$ : Concentration of infectious microorganisms after exposure to UV light

Fig. 2 shows the refined grids were imposed in the vicinity of the perforated plate and other separation zone. For a mesh convergence study, the effect of mesh density on pressure drop was evaluated. As a result of a mesh convergence study, it was decided to use a grid of 9.39 million because there was almost no effect in pressure loss between inlet and outlet, even if the number of grids was increased. The dose distribution histogram depends on the flow characteristics of the fluid. Therefore, it is important to know the flow characteristics in the reactor. Fig. 4(a) shows the streamline of sea water, which is well distributed after passing the perforated plate. In addition, the fluid velocity inside the reactor near the outlet is faster than the far side. The contours in Fig. 4(b) show the velocity distribution of the UV reactor at cross-section. As shown in the 
color of the contour, indeed, the velocity increases when passing through the perforated plate due to the narrow channel. However, the velocity distribution after the perforated plate is uniform. In addition, the velocity increases again near the lamps due to the same reason. This non-uniformity of flow stream causes a difference in the cumulative dose that each microorganism moves along the stream line. That is, the microorganisms moving in the region having a low flow rate are irradiated with UV light for a relatively long time, and the microorganisms exercising at the region having a high flow rate are irradiated with UV light for a short time [7].

Prior to the dose calculation, particle independence test was performed in the reference case (power is $100 \%$, flow rate is 200 $\mathrm{m}^{3} / \mathrm{h}$, and UVT is $70 \%$ ). This study was conducted to determine the appropriate number of particles. As a result, it can be confirmed that the particle dose rate is stabilized when the number of particles becomes more than about 20,000 as shown in the following Fig. 5 . Therefore, if the injection amount is more than 20,000, the error mean dose is less than $0.2 \%$ even if the number of particles is increased more than 7 times. Herein, 20,795 particles were used for the dose calculation. A remaining issue is how to calculate the RED which describes the level of disinfection of the UV reactor. We can obtain the probability (\%) divided by the total number of particles at the certain dose $\left(\mathrm{J} / \mathrm{m}^{2}\right)$. As expressed in Eq. (5), total log inactivation was obtained by correlation between the probability and the log inactivation of organism.

Total log inactivation of Tetraselmis : $-\sum_{\min }^{\max } \log \left(A \cdot 10^{-B}\right)$

Where A is the probability (\%), B is the log inactivation of Tetraselmis $\left(\log \left(\mathrm{N} / \mathrm{N}_{0}\right)\right)$, min is the minimum value of dose $\left(\mathrm{J} / \mathrm{m}^{2}\right)$, and max is the maximum value of dose $\left(\mathrm{J} / \mathrm{m}^{2}\right)$.

The total log inactivation derived from the Eq. (5) is then related to the UV dose response curve obtained from the CBT. This dose is termed the reduction equivalent dose (RED).

We concluded that RED was calculated by following formula.

$$
\begin{gathered}
\operatorname{RED}\left(\mathrm{J} / \mathrm{m}^{2}\right)= \\
500 \log \left(\mathrm{N}_{0} / \mathrm{N}\right) \times \text { Total } \log \text { inactivation of Tetraselmis }
\end{gathered}
$$

We intuitionally know that the RED can be changed with the flow rate, UVT, and lamp output since UV disinfection level is highly dependent on the RED [16]. Undoubtedly, RED increased with decreased flow rate and increased with increased UVT. As shown in Table 1, we figured out that calculated RED is $771 \mathrm{~J} / \mathrm{m}^{2}$ at the design condition (flow is $360 \mathrm{~m}^{3} / \mathrm{h}$, UVT is $70 \%$, and power is $100 \%$ ), which is higher than required RED of $750 \mathrm{~J} / \mathrm{m}^{2}$. It implies that the Tetraselmis can safely be inactivated over 99.9\%. Table 2 shows the calculated RED with different UVT at flow $200 \mathrm{~m}^{3} / \mathrm{h}$. When the lamp output is set to $100 \%$, we can expect the RED to be over $750 \mathrm{~J} / \mathrm{m}^{2}$ when the UVT is between 50 and $60 \%$. On the other hands, RED to be over $750 \mathrm{~J} / \mathrm{m}^{2}$ when the UVT is between 60 and $70 \%$ at flow $360 \mathrm{~m}^{3} / \mathrm{h}$ (Table 1). On the aspect of engineering, UV reactor is possible to be operated where UVT is ranged between $60 \%$ and $70 \%$ at flow rate of $360 \mathrm{~m}^{3} / \mathrm{h}$. If BWTS should be operated in high turbidity regions, the operators can reduce the flow rate to meet the design RED. Therefore, UV reactor can be continuously operated where UVT is ranged between $50 \%$ and $60 \%$ at flow rate of $200 \mathrm{~m}^{3} / \mathrm{h}$.

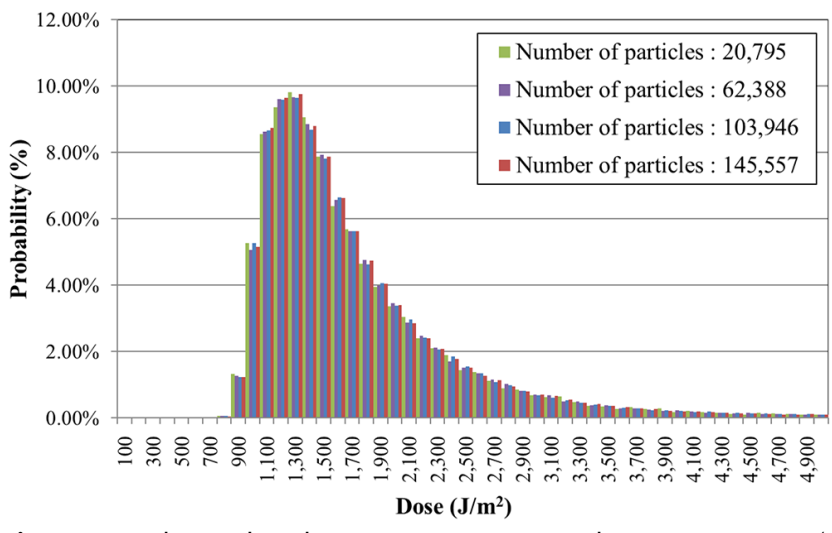

Fig. 5. Cumulative dose histogram at reactor outlet. Power is $100 \%$, flow rate is $200 \mathrm{~m}^{3} / \mathrm{h}$, and UVT is $70 \%$.

\begin{tabular}{|c|c|c|c|c|c|}
\hline Power UVT & $50 \%$ & $60 \%$ & $70 \%$ & $\mathbf{8 0} \%$ & $90 \%$ \\
\hline $40 \%$ & 178 & 242 & 353 & 535 & 1,001 \\
\hline $70 \%$ & 288 & 393 & 584 & 894 & 1,677 \\
\hline $100 \%$ & 387 & 531 & 771 & 1,236 & 2,310 \\
\hline
\end{tabular}

Table 1. Simulated RED with Different UVT at Flow $360 \mathrm{~m}^{3} / \mathrm{h}$

\begin{tabular}{|c|c|c|c|c|c|}
\hline Power UVT & $50 \%$ & $60 \%$ & $70 \%$ & $\mathbf{8 0} \%$ & $90 \%$ \\
\hline $40 \%$ & 291 & 393 & 590 & 896 & 1,691 \\
\hline $70 \%$ & 462 & 634 & 966 & 1,489 & 2,796 \\
\hline $100 \%$ & 612 & 854 & 1,321 & 2,050 & 3,806 \\
\hline
\end{tabular}

Table 2. Simulated RED with Different UVT at Flow $200 \mathrm{~m}^{3} / \mathrm{h}$

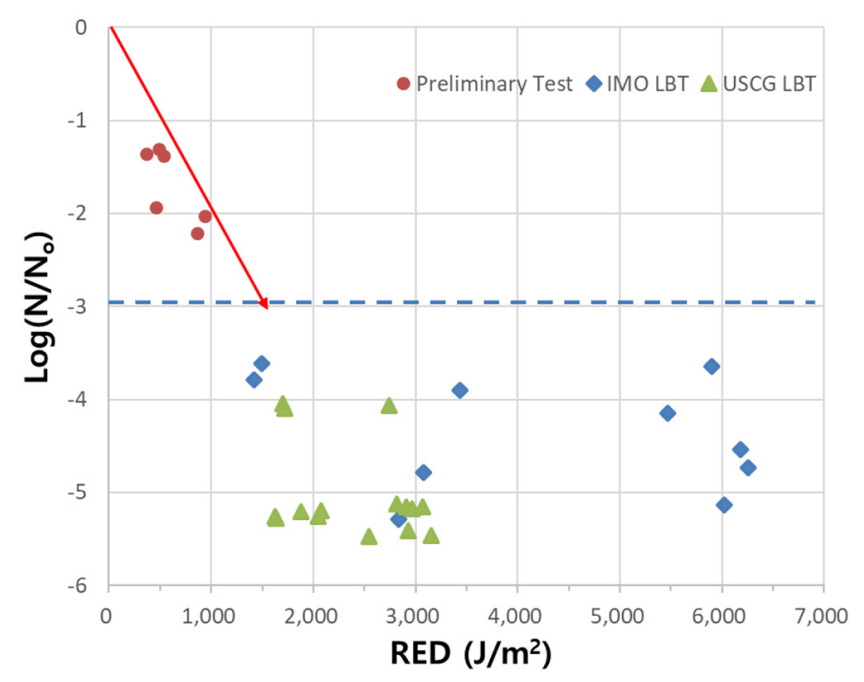

Fig. 6. Log inactivation versus calculated RED using Tetraselmis. The red arrow indicates $500 \log \left(\mathrm{N} / \mathrm{N}_{0}\right)$.

Finally, it is important to verify the performance in a real scale UV reactor through $\mathrm{BE}$ test. Please remind that the required RED of $1,500 \mathrm{~J} / \mathrm{m}^{2}$ is necessary to treat the ballast water twice in the UV reactor during ballasting and deballasting. Fig. 6 summarized IMO, USCG LBT and preliminary testing results. And the RED was estimated by interpolation method because no UVT adjustment 
can accurately be done during LBT. Both IMO and USCG LBT, the ballast water is filtered and subsequently sterilized through the UV reactor. However, we can exclude the influence of the filter since the filter was not responsible for the reduction of Tetraselmis. Significant reduction of live tetraselmis was only observed through UV treatment [17]. After evaluating the inactivation ratio of Testraselmis, the log inactivation versus RED is plotted in Fig. 6. The LBT results give us useful information to validate the performance of the real scale UV reactor. We recognize that 3-log inactivation of Tetraselmis was satisfied when the RED was above $1,500 \mathrm{~J} / \mathrm{m}^{2}$. In other words, below blue dotted line indicates the efficacy rate was over $99.9 \%$. In addition, when the RED is below $1,500 \mathrm{~J} / \mathrm{m}^{2}$, log inactivation ratio should comply with the red arrow line to meet the design value. We found that when RED was lower than $1,500 \mathrm{~J} / \mathrm{m}^{2}$, that is, the $\log$ inactivation value was under the red arrow, which implies that it shows a better efficacy performance than the design of log inactivation.

\section{Conclusions}

The calculated RED is obtained from the results of CBT with dose estimates from numerical modeling. The calculated RED is 771 $\mathrm{J} / \mathrm{m}^{2}$ at the design condition. We found that the UV reactor is well designed because the calculated RED is higher than the required RED $\left(750 \mathrm{~J} / \mathrm{m}^{2}\right)$ for Tetraselmis inactivation. Through BE tests of LBT, 3-log Tetraselmis inactivation was satisfied when the RED was higher than that of $1,500 \mathrm{~J} / \mathrm{m}^{2}$. In addition, when the RED is below $1,500 \mathrm{~J} / \mathrm{m}^{2}$, the log inactivation value was under the slope of $500 \log \left(\mathrm{N} / \mathrm{N}_{0}\right)$. It implies that it shows a better efficacy performance than the design value. In the aspect of engineering, UV reactor is possible to be operated where UVT is ranged between $60 \%$ and $70 \%$ at flow rate of $360 \mathrm{~m}^{3} / \mathrm{h}$. And UV reactor can be operated where UVT is ranged between $50 \%$ and $60 \%$ at flow rate of 200 $\mathrm{m}^{3} / \mathrm{h}$. We hope this manuscript will give insight into the design of the real scale UV reactor for BWTS.

\section{Author Contributions}

J.Y.J. (Senior Researcher) and G.J.J. (Senior Researcher) designed UV reactor and conducted the experiment. I.S.O. (Senior Researcher) performed the CFD modeling and T.J.R. (Senior Researcher) conducted the experiment.

\section{References}

1. International Maritime Organization (IMO). Ballast water management - the control of harmful invasive species. Copyright 2019 International Maritime Organization (IMO). Available from: http://www.imo.org/en/MediaCentre/HotTopics/BWM/Pages/ default.aspx

2. International Maritime Organization (IMO). International Convention for the Control and Management of Ship's Ballast Water and Sediments (BWM). Available from: http://www.im-
o.org/en/About/Conventions/ListOfConventions/Pages/Internat ional-Convention-for-the-Control-and-Management-ofShips'-Ballast-Water-and-Sediments-(BWM).aspx

3. United Stated Code of Federal Regulations (CFR). CFR Part 151, Subpart D-Ballast water Management for Control of Nonindigenous Species in Waters of the United States. § 151.2030 Ballast water discharge standard (BWDS)

4. Tsolaki E, Diamadopoulos E. Technologies for ballast water treatment: A review. J. Chem. Technol. Biotechnol. 2010;85:19-32.

5. Batista WR, Fernandes FC, Lopes CC, Lopes RSC, Miller W, Ruiz G. Which ballast water management system will you put aboard? Remnant anxieties: A mini-review. Environments 2017;4:1-12.

6. Hess-Erga OK, Moreno-Andrés J, Enger Ø, Vadstein O. Microorganism in ballast water: Disinfection, community dynamics, and implications for management. Sci. Total Environ. 2019;657:704-716.

7. Chen JY, Deng B, Kim CN. Computational fluid dynamics (CFD) modeling of UV disinfection in a closed-conduit reactor. Chem. Eng. Sci. 2011;66:4983-4990.

8. Cosman J, Fraser J, Latimer G, Gerardi-Fraser J. Validation protocol for performance testing and scaling ballast water treatment systems employing UV disinfection and filtration. Proceedings of the IMO-WMU Research and Development Forum, 26-27 January, 2010; Sweden, p. 261-273.

9. ETV protocol, Generic protocol for the verification of ballast water treatment technology. U.S. Environmental Protection Agency (EPA) 2010.

10. Lundgreen K, Holbech H, Pedersen KL, et al. Use of standard test organisms for sound validation of UV-based ballast water treatment systems. Mar. Pollut. Bull. 2019;144:253-264.

11. Sun Z, Blatchley III ER. Tetraselmis as a challenge organism for validation of ballast water UV systems. Water Res. 2017;121:311-319.

12. Pyo TS, Cheon SG, Park DW, Choi SK, Kim SY, Kil GS. Design and Fabrication of a Ballast Water Treatment System Using UV Lamps. J. Korean Soc. Mar. Eng. 2009;33:952-958.

13. Olsen RO, Lindivat M, Larsen A, Thuestad G, Hoell IA. Incubation in light versus dark affects the vitality of UV-irradiated Tetraselmis suecica differently: A flow cytometric study. Mar. Pollut. Bull. 2019;149:1-8.

14. Olsen RO, Hoffmann F, Hess-Erga OK, Larsen A, Thuestad G, Hoell IA. Ultraviolet radiation as a ballast water treatment strategy: Inactivation of phytoplankton measured with flow cytometry. Mar. Pollut. Bull. 2016;103:270-275.

15. Park KY, Kim YG, Jung GJ, Han KH, Kapmann M, Wang J. Development of a new UV-Treatment System for Ballast Water Application. International Conference on Ballast Water Management, 16-17 October, 2008; Singapore.

16. Kim H, Bak J, Lee K, Cho J. A study on CFD Methodology of the performance Prediction for the UV Disinfection Reactor. KSFM 2014;17:44-51.

17. Pazouki K, Carney KJ, Delany J, Mesbahi E. Comparison of land-based test setups for a ballast water management system. Int. J. Marit. Eng. 2011;153:1-12. 